\title{
Primary adenocarcinoma of the seminal vesicles. A review of the literature
}

\author{
Ioannis Katafigiotis ${ }^{1}$, Stavros Sfoungaristos ${ }^{2}$, Mordechai Duvdevani ${ }^{2}$, Panagiotis Mitsos ${ }^{1}$, \\ Eleni Roumelioti ${ }^{1}$, Konstantinos Stravodimos ${ }^{1}$, Ioannis Anastasiou ${ }^{1}$, Constantinos A. Constantinides ${ }^{1}$ \\ 1 1st University Urology Clinic Laiko Hospital, Athens, Greece; \\ ${ }^{2}$ Hebrew Hadassah University Medical Center, Jerusalem, Israel.
}

\begin{abstract}
Summary Primary adenocarcinoma of the seminal vesicles (SV) are extremely rare and approximately only 60 cases have been reported in the literature. Due to the lack of specific symptoms the patients often present in an advanced stage of their disease. The only clinical examination that can indicate the presence of a neoplasm in the SVs is the digital rectal examination (DRE). Serum prostatic specific antigen (PSA) and prostate specific acid phosphatase (PAP) are usually normal in patients with primary adenocarcinoma of the SV and only CA-125 can be proved a useful blood biomarker contributing to the diagnosis and the follow up of the SV adenocarcinoma. Computed tomography (CT) and magnetic resonance imaging (MRI) and FDG-PET/CT have been used for the diagnosis and the staging of the SV adenocarcinoma. Various combinations of radical surgery, radiotherapy androgen deprivation therapy and chemotherapy have been proposed for the management of the disease but the prognosis is poor and the mean survival is two years after the diagnosis.
\end{abstract}

KEY WORDS: Primary adenocarcinoma of the seminal vesicles; Seminal vesicle cancer; Primary neoplasm of the seminal vesicle.

Submitted 27 October 2015; Accepted 5 December 2015

\section{INTRODUCTION}

Primary adenocarcinoma of the seminal vesicles (SV) are extremely rare and approximately only 60 cases have been reported in the literature (1). Due to the lack of specific symptoms the diagnosis and differentiation of a SV adenocarcinoma from neoplasms of the anatomic vicinity like the prostate, bladder and rectum that infiltrate secondarily the SVs is usually difficult (2). We present a comprehensive review of the literature.

\section{MATERIAL AND METHOdS-AEARCH ATRATEgY}

Two different Pubmed-based search strategies were followed in order to acquire the necessary data for a complete review. The first search was "Primary adenocarcinoma of the seminal vesicles" and 87 papers was the original result. From these 87 papers 21 papers-references were chosen and constituted the main base of the review; 66 papers were excluded either because they were irrelevant to the subject, either because no abstract was avail- able. Also papers that were referring to primary cancer of the seminal vesicle but to histology types other than adenocarcinoma were excluded from the main text but were used to the formation of Table I in the classification of the histology types. An additional search was made with "Primary seminal vesicle carcinoma" being the key words; 185 papers was the result and only 2 were chosen. The majority of the papers excluded were either the same papers as in the first search either irrelevant to the subject. After the final selection 23 papers were chosen on which the review was based. One additional reference (reference 3) was not present in the Pubmed search but was traced from another paper and was used only for the historical aspect of the subject since it was the first paper published referring to the subject.

\section{Discussion}

\section{Epidemiology}

Primary carcinomas of the SVs are extremely rare and the first case presented in the literature was by Lyons in 1925 (1, 3). Although primary adenocarcinomas of the SVs are rare it isn't very easy to record with precision the exact number of the cases recorded in the literature. Following the papers published year by year in the literature, in 2000 only 48 cases were recorded (4), in 2006 49 cases (5), 50-51 cases in $2011(6,7)$, and 5 more since then rising the total number of cases to 55-56 ( 1 , $8-11)$. The mean patient age at diagnosis is 62 years old (range: 13-90 years) (9).

\section{Histology types}

Primary adenocarcinoma is the most common neoplasm of the SVs. Other histology types of the primary neoplasms of the SVs that have been recorded in the literature are sarcomas, squamous cell carcinoma, yolk salk tumor, neuroendocrine carcinoma, paraganglioma, epithelial stromal tumors, lymphoma (Burkitt, B-Cell), extragastrointestinal stromal tumor (EGIST), myxoid solitary fibrous tumor, and seminoma peripheral primitive neuroectodermal tumor (PPNET) (Table 1). Benign tumors of the SVs are even rarer (5) and the reported histology types in the literature are cystic dysplasia, fibroepithelial tumor, leiomyoma, cystadenoma and Schwannoma (Table I). 


\section{Table 1.}

Histology types.

\begin{tabular}{|c|c|c|}
\hline $\begin{array}{l}\text { Primary neoplasms } \\
\text { of the SVs }\end{array}$ & Histology type & References \\
\hline Malignant & $\begin{array}{l}\text { 1. Squamous cell } \\
\text { 2. Yolk salk } \\
\text { 3. Neuroendocrine } \\
\text { 4. Paraganglioma } \\
\text { 5. Epithelial stromal tumor } \\
\text { 6. Extragastrointestinal stromal tumor } \\
\text { 7. Myxoid solitary fibrous tumor } \\
\text { 8. Seminoma } \\
\text { 9. Lymphoma } \\
\text { - Burkitt } \\
\text { - B Cell } \\
\text { 10. Sarcomas } \\
\text { - Leiomyosarcoma } \\
\text { - Angiosarcoma } \\
\text { - Extraskeletal osteosarcoma } \\
\text { - Cauvin C,et al. } 2011 \\
\text { - Chang K, et al. } 2014 \\
\text { - Choi JD, et al. } 2011 \\
\text { 11. Peripheral primitive neuroectodermal tumor (PPNET) }\end{array}$ & $\begin{array}{l}\text { Wang J, et al. } 2013 \\
\text { Tabata K, et al. } 2001 \\
\text { Dong Yao, et al. } 2012 \\
\text { Kreiner B, et al. } 2010 \\
\text { Alvarengal CA, et al. } 2012 \\
\text { Hoshi A, et al. } 2006 \\
\text { Song W, et al, } 2012 \\
\text { Wei YC, et al. } 2006 \\
\text { Adachi Y, et al. } 1991 \\
\text { - Ouyang J, et al. } 2009 \\
\text { - Jiang Zhu, et al. } 2011 \\
\end{array}$ \\
\hline Benign & $\begin{array}{l}\text { 1. Cystic dysplasia } \\
\text { 2. Fibroepithelial tumor } \\
\text { 3. Leiomyoma } \\
\text { 4. Cystadenoma } \\
\text { 5. Schwannoma }\end{array}$ & $\begin{array}{l}\text { Domínguez DM, et al. } 1999 \\
\text { Zanetti GR, et al. } 2003 \\
\text { Gentile AT, et al. } 1994 \\
\text { Lee CB, et al. } 2006 \\
\text { Arun G, et al. } 2014\end{array}$ \\
\hline
\end{tabular}

neoplasm the majority of the cases in the literature refer to a large irregular mass above the prostate (13). Usually the mass is non tender and immobile with a hard elastic composition $(5,9,8,13)$. Even though an abnormal DRE constitutes an integral part of the diagnosis, up to $30 \%$ of patients have no abnormal findings during DRE (9).

\section{c) Blood biomarkers -Table 2}

Serum prostatic specific antigen (PSA) and prostate specific acid phosphatase (PAP) are usually normal in patients with primary adenocarcinoma of the SVs $(1,9$, $6,13)$. Also in our case the patient had a normal PSA of 1.5 ng/ml. Apart from the PSA also CA-19.9 has been reported to be normal in patients with primary adenocarcinoma of the SVs $(1,9)$. Special consideration must be taken for the other two biomarkers CEA and CA-125. Normal serum CEA can be interpreted as absence of invasion of a colon carcinoma (9). Although usually

\section{Diagnosis}

From 1956 already Dalgaard and Giertson described the following criteria for the diagnosis of a primary adeonocarcinoma of a SVs: the tumor should be a macro- and microscopically verified carcinoma, localized exclusively or mainly to the seminal vesicle; there must be no other primary carcinoma in the body; and the tumor should preferably be a papillary adenocarcinoma that resembles the architecture of the non-neoplastic seminal vesicle (4, 12). Nevertheless these criteria are referring mainly to the final specimen of the surgical resection 4 and the presurgical diagnosis is a difficult task and the differential diagnosis from the primary adenocarcinomas of the prostate, bladder and colon that infiltrate the SVs is even more difficult $(6,9,11)$.

\section{a) Symptoms}

Due to the lack of specific symptoms the patients often present in an advanced stage of their disease (13). All the data in the literature refer to non-specific symptoms from the genitourinary and the gastrointestinal tract $(1,5)$. The main symptoms from the genitourinary system can be dysuria, frequency, hematuria, hematospermia and bladder outlet obstruction $(1,5,9)$. From the gastrointestinal tract the main symptoms can be constipation and intestinal bleeding $(8,11)$. Other symptoms that have been reported are painful sensation in the pelvis and perineum and general symptoms like appetite loss $(1,8)$.

b) Clinical examination-Digital rectal examination (DRE)

The only clinical examination that can indicate the presence of a neoplasm in the SVs is the DRE. Although there is no unanimous description of the findings of a DRE in SV
CEA is normal in primary adenocarcinoma of the SVs, increased serum CEA levels are seen in rare cases $(1,9$, 13). CA-125 is a useful biomarker in SV adenocarcinoma since there are reports of tumors producing CA-125 elevating the levels of the marker in $\operatorname{serum}(9,14)$. CA-125 is a useful biomarker not only for the diagnosis but also for the follow-up of the patient since it can be decreased even to normal value after the treatment or rise again up to 6 months before the occurrence of clinical metastases (8, 14). Finally there is a reference of an elevated CA15-3 in a patient with primary SV adenocarcinoma (9).

\section{Table 2.}

Profile of blood biomarkers in primary adenocarcinoma of the seminal vesicles.

\begin{tabular}{|ll}
\hline PSA & Normal \\
\hline CA-19.9 & Normal \\
\hline CA-125 & Usually elevated but could be normal \\
\hline CA 15-3 & Normal rarely elevated \\
\hline
\end{tabular}

\section{d) Imaging-biopsy}

Computed tomography (CT) and magnetic resonance imaging (MRI) have been widely used for the depiction of a SV neoplasm $(1,9,13)$. Various descriptions of a SV mass during a CT have been reported in the literature from a solid mass originating from the SVs mildly enhancing to a lesion with an hypodense central area presenting peripheral contrast enhancement $(1,8,13)$. MRI imaging can depict a high signal-intensity fluid in the SV (T1-weighted), a papillary mass (T2-weighted), or 
even a malignant cystic-necrotic lesion with irregular solid components $(9,13)$. Nevertheless MRI can also depict the anatomy and the extent of the SV neoplasm contributing to the preoperative evaluation of the case $(7,15)$. FDG-PET/CT has also been used for the diagnosis the staging and the assessment of the treatment response $(1,8)$. FDG-PET/CT usually confirms radiopharmaceutical uptake in the primary SV lesion $(1,8)$. Transrectal ultrasonography has been recommended as the first imaging method because can contribute both to the depiction of the mass and a simultaneous biopsy that will set the diagnosis $(1,9)$. A solid mass or a cystic lesion with the inclusion of a solid mass is the usual depiction at transrectal ultrasonography $(13,16)$. Cystoscopy is usual negative and has been reported to be normal up to $20 \%$ of patients and it isn't the standard method used for the depiction and the diagnosis of a SV $(1,9)$. Colonoscopy has been proposed for the exclusion of a primary rectal tumor $(8,17)$.

\section{e) Immunohistochemical analysis and differential diagnosis (Table 3)}

Immumohistochemical analysis can play a vital role both for the diagnosis and the differentiation of a SV adenocarcinoma from prostate, bladder and the colorectal carcinomas $(1,4,6,9)$. All the data in the literature report an absence of staining both for prostate specific antigen (PSA) and prostate-specific acid phosphatase (PAP) of the SV adenocarcinoma contributing in the differentiation from a prostatic adenocarcinoma $(1,4)$. CA-125 a large, highly glycosylated glycoprotein, though is used as a marker of mullerian differentiation, can even show a paradoxical immunoreactivity to a well-differentiated papillary SV adenocarcinoma that is of a wolffian duct origin $(4,6)$. CA- 125 can help in the differentiation of a SV adenocarcinoma from prostatic, bladder and rectal tumors, since these carcinomas are usually negative (4). CA-125 immunoreactivity alone may be not sufficient for the diagnosis of a SV adenocarcinoma, since other tumors that can express CA-125, although less frequently, like breast, pancreatic, lung, colon carcinomas, primary serous carcinoma of the peritoneum, clear cell adenocarcinoma of the bladder and metastases especially from a pancreatic-biliary carcinoma and clear cell adenocarcinoma of the bladder must be ruled out $(4,6)$. At the same time there are cases in the literature of SV adenocarcinomas especially with a poor differentiation that are CA- 125 negative $(1,4)$. Simultaneously, poorly differentiated prostatic adenocarcinomas can be negative to PSA and PAP although only in $1 \%$ to $2 \%$ of cases, constituting the use of additional markers necessary (4). The profile of cytokeratins 7 and 20 can help further in the differential diagnosis of a SV adenocarcinoma since CK7 is usually positive and CK20 negative $(1,4,6)$. The combination of PSA, PAP, CA-125, CK7, and CK20 markers can help in the discrimination of a SV adenocarcinoma from a bladder, prostatic and colon adenocarcinoma, since the PSAnegative, PAP-negative, CK20-negative and CK7 and CA125 positive profile of a SV adenocarcinoma is unique (4). Also the negative immunoreactivity of the CDX-2 of a SV adenocarcinoma can contribute to the discrimination form a colon cancer $(1,6)$. CEA should be negative
Table 3.

Profile of Immunohistochemical markers in primary adenocarcinoma of the seminal vesicles.

\begin{tabular}{|ll|}
\hline PSA & Negative \\
\hline PAP & Negative \\
\hline CA-125 & Positive \\
\hline CK 7 & Positive \\
\hline CK20 & Negative \\
\hline
\end{tabular}

in a primary SV adenocarcinoma but there are many cases of CEA positive or focally positive SV adenocarcinomas reported in the literature $(1,4,9,17)$.

\section{Metastases}

Primary adenocarcinoma of the SVs can invade both the adjacent organs and give distant metastases. Invasion to the rectum leads to rectal bleeding $(8,11)$. Due to the anatomic vicinity. primary adenocarcinoma can invade both the prostate and the bladder $(18,19)$. Lymph node metastases to the pelvis, to the infrarenal para-aortic nodes, to the cervical chain and the mediastinal lymph nodes have been reported $(6,9,10)$. Apart from the lymph nodes, metastases to the lungs and the liver are common $(2,6,8,9,11)$. Skeletal metastases with severe pain have also been reported to one case in the literature (20). There is also a rare case of an isolated penile metastasis from seminal vesicle adenocarcinoma (2). Finally a case of a disseminated carcinoma with metastases to lymph nodes, pleura, pericardium, liver, heart, and lung revealed to an autopsy of a patient died from a primary adenocarcinoma of the seminal vesicles (6).

\section{Treatment}

Although local excision of the mass-SVs has been proposed the majority of the data in the literature propose a radical surgery as a part of a multimodal approach combined with hormone therapy, radiation therapy and chemotherapy. Local excision, whenever possible due to the adhesions with the surrounding tissues, has been proposed as a conservative surgical approach again as a part of a multimodal approach $(9,20)$. In one case the local excision couldn't achieve a complete removal of the tumor leaving residual mass to the bladder and the rectum9. Radical surgery and the extent of the necessary limits are not well defined and various operations have been performed in order to achieve a complete resection of the tumor $(10,11,21,22)$. Radical prostatectomy, radical cystoprostatectomy, partial cystectomy and total pelvic exenteration with ileal conduit for urinary diversion and a sigmoid colostomy, combined with pelvic lymphadenectomy are the various radical operations suggested in the literature $(1,0,11,13,21)$.

The best chance of extended survival seems to be the combination of a radical surgery with clear margins combined with adjuvant hormone therapy, or radiotherapy or both $(10,21-23)$. Hormone therapy has been proposed as an adjunct therapy to surgery or to radiotherapy both for the local control of the tumor and the management of the metastases $(2,8,20,22,23)$.

In the hormone manipulation and the achievement of androgen deprivation, estrogens and bilateral orchiecto- 
my and have also been used $(2,20,24)$. Radiotherapy to the whole pelvis combined with antiandrogen blockade without surgery has been proposed as a sole therapy but the patient developed liver metastases 19 months later receiving two cycles of docetaxel and cisplatin chemotherapy and finally dying of his disease 22 months after the diagnosis (8). Radiotherapy has also been proposed also for the management of local lymph node metastases, with pelvic irradiation combined with chemotherapy and for the management of residual mass after the operation $(1,9,10)$. As an adjuvant therapy for the management of residual mass a total dose of $50 \mathrm{~Gy}$ in 2 Gy fractions has been proposed (9). Hormone therapy or chemotherapy or the combination of both modalities have been used for the management of distal metastases $(1,2,8,9,20)$.

Various combinations of chemotherapy regimens have been used, like docetaxel and cisplatin (2 cycles) for the management of liver metastases, cisplatin and 5-FU (6 cycles) for pulmonary lesions, and taxol with caroplatinum (6 Cycles) for a left transverse perineal muscle lesion $(1,8,9)$. In one case with skeletal metastases an one year estrogen therapy resulted the disappearance of the metastases and the survival of the patient for at least for two years (20). Finally the combination of six cycles of 5-fluorouracil, leucovorin, and oxaliplatin chemotherapy with bilateral orchiectomy to a patient with an isolated metastasis to penis, resulted the clinical improvement of the patient and the regression of penile swelling and seminal vesicle mass, but the patient developed later lung metastases and died (2).

\section{Prognosis}

Prognosis of a primary vesicle adenocarcinoma of the SVs is general considered poor due to delayed diagnosis and approximately the $95 \%$ of the patients die in less than three years $(9,11,22,23)$. Although there is a case of a patient dying three months postoperatively and two cases of a prolonged survival of 3 years and 4 months in one case and 5 years in the other, the majority of the cases in the literature refer to an approximately 2 years survival after the diagnosis $(8,10,11,13,18,20)$.

\section{Follow-up}

The main goal of the follow up of the patient with a primary vesicle adenocarcinoma is the detection of metastases. A combination of clinical examination, serum tumor markers, CT (chest and abdomen) MRI and [18F]FDG-PET/CT has been proposed for the follow up $(1,8,9,22)$. CA- 125 can be used for the detection of the recurrence and the clinical course of the disease $(8,15$, 22). CA- 125 elevation in some cases precede up to 6 months before the emergence of the metastases (8).

Suspicious lesions to the surgical bed to patients with possible residual mass or positive surgical margins can be detected with the use of MRI (9). CT scan of the chest and the abdomen combined with clinical examination and serum markers every 3 months for the first year and every 6 months thereafter have been proposed as a follow up scheme (1). [18F]FDG-PET/CT can help in the clarification of suspicious lesions depicted in the CT $(1,9)$.
Lesions to soft tissues depicted to [18F]FDG-PET/CT can be further clarified with the use of an MRIl. Finally ultrasound guided cytology has been used during the follow up for the confirmation of a metastasis (1).

\section{Risk factors and bilateral primary SV adenocarcinomas}

Although may not be easy to locate the SV from the cancer originates, due to the volume of the mass, there are cases that clearly declare the bilateral origin of the primary vesicle adenocarcinoma $(10,18)$. The bilateral primary SV adenocarcinoma is considered even more rare (18). Considering the risk factors or predisposition for the occurrence of a primary SV adenocarcinoma the presence of a renal agenesis or dysgenesis has been reported to be a possible aggravating factor $(10,13)$.

The renal agenesis or dysgenesis seems to contribute either to a ipsilateral or a bilateral SV adenocarcinoma $(10,13)$. The proposed pathway for the development of a SV adenocarcinoma is the chronic stimulation of the ectopic ureter's secretion (13)

\section{REFERENCES}

1. Sollini M, Silvotti $M$, Casali $M$, et al. The role of imaging in the diagnosis of recurrence of primary seminal vesicle adenocarcinoma. World J Mens Health. 2014; 32:61-5.

2. Thyavihally YB, Tongaonkar HB, Gupta S, Gujral S. Primary seminal vesicle adenocarcinoma presenting as isolated metastasis to penis responding to chemotherapy and hormonal therapy. Urology. 2007; 69:778.e1-3.

3. Lyons O Primary carcinoma of the left seminal vesicle. J Urol. $1925 ; 13: 477$.

4. Ormsby AH, Haskell R, Jones D, Goldblum JR. Primary seminal vesicle carcinoma: an immunohistochemical analysis of four cases. Mod Pathol. 2000; 13:46-51

5. Hoshi A, Nakamura E, Higashi S, et al. Epithelial stromal tumor of the seminal vesicle. Intern J Urol. 2006; 13:640-642

6. Stenzel P, Wettach G, Leroy X. Primary seminal vesicle carcinoma. Intern J Surg Path. 19(3)401-404.

7. Angulo JC, Romero I, Cabrera P, et al. Vesiculectomy with laparoscopic partial prostatectomy in the treatment of primary adenocarcinoma of the seminal vesicle with carcinomatous transformation of the ejaculatory duct. Actas Urol Esp. 2011; 35:304-9.

8. Mizuno N, Fujikawa N, Hayashi N, et al. A case of primary seminal vesicle cancer detected by FDG-PET/CT. Nihon Hinyokika Gakkai Zasshi. 2012; 103:704-7.

9. Eken A, Izol V, Aridogan IA, et al. An unusual cause of hematospermia: Primary adenocarcinoma of the seminal vesicle. Can Urol Assoc J. 2012;6:E259-E262.

10. Campobasso D, Fornia S, Ferretti S, et al. Primary bilateral seminal vesicle carcinoma: description of a case and literature review. Int J Surg Pathol. 2012; 20:633-5.

11. Kinjo T, Nonomura D, Yamamoto Y, et al. Primary adenocarcinoma of the seminal vesicle difficult to differentiate from rectal carcinoma : a case report. Hinyokika Kiyo. 2013; 59:597-601.

12. Dalgaard JB, Giertson JC. Primary carcinoma of the seminal vesicle: case and survey. Acta Pathol Microbiol Scand. 1956; 39:255-67. 
13. Lee BH, Seo JW, Han YH, et al. Primary mucinous adenocarcinoma of a seminal vesicle cyst associated with ectopic ureter and ipsilateral renal agenesis: a case report. Korean J Radiol. 2007; 8:258-61.

14. Ohmori T, Okada K, Tabei R, et al. CA125-producing adenocarcinoma of the seminal vesicle. Pathol Int. 1994; 44:333-7.

15. Navallas M, Vargas HA, Akin O, et al. Primary seminal vesicle adenocarcinoma. Clin Imaging. 2011; 35:480-2.

16. Al-Saeed O, Sheikh M, Kehinde EO, Makar R. Seminal vesicle masses detected incidentally during transrectal sonographic examination of the prostate. J Clin Ultrasound. 2003; 31:201-6.

17. M. Tarján, I. Ottlecz, T. Tot. Primary adenocarcinoma of the seminal vesicle. Indian J Urol. 2009; 25:143-145.

18. Ormsby AH, Haskell R, Ruthven SE, Mylne GE. Bilateral primary seminal vesicle carcinoma. Pathology. 1996; 28:196-200.
19. Tanaka T, Takeuchi T, Oguchi K, et al. Primary adenocarcinoma of the seminal vesicle. Hum Pathol. 1987; 18:200-2.

20. Kindblom LG, Pettersson G. Primary carcinoma of the seminal vesicle. Case report. Acta Pathol Microbiol Scand A. 1976; 84:301-5.

21. Möhring C, Bach P, Kosciesza S, Goepel M. A primary adenocarcinoma of the seminal vesicles. Case report of a rare malignancy. Urologe A. 2008; 47:616-9.

22. Thiel R, Effert P. Primary adenocarcinoma of the seminal vesicles. J Urol. 2002; 168:1891-6.

23. Benson RC Jr, Clark WR, Farrow GM. Carcinoma of the seminal vesicle. J Urol. 1984; 132:483-5.

24. RODRIGUEZKEES OS. Clinical improvement following estrogenic therapy in a case of primary adenocarcinoma of the seminal vesicle. J Urol. 1964; 91:665-70.

\section{Correspondence}

Ioannis Katafigiotis, MD, MLS, PhD, Resident in Urology (Corresponding Author) katafigiotis@yahoo.com

Panagiotis Mitsos, MD Resident In Urology

mitsospanagiotis@yahoo.com

Eleni Roumelioti, Finance and Statistics

eleniroum81@gmail.com

Konstantinos Stravodimos, MD, PhD, FEBU Associate Professor in Urology

kgstravod@yahoo.com

Ioannis Anastasiou, MD, PhD, FEBU Assistant Professor in Urology

ekati2@otenet.gr

Constantinos A. Constantinides, MD, PhD, FEBU Professor in Urology

ckonstan@med.uoa.gr

$1^{\text {st }}$ University Urology Clinic Laiko Hospital

Agiou Thoma 17 - 11527 Athens, Greece

Stavros Sfoungaristos, $\mathrm{MD}, \mathrm{PhD}$

sfoungaristosst@gmail.com

Mordechai Duvdevani, MD, Associate Professor in Urology

moti_duv@yahoo.com

Hebrew Hadassah University Medical Center

Jerusalem, Israel 